\title{
ERR1- and PGC1 $\alpha$-associated mitochondrial alterations correlate with pan-cancer disparity in African Americans
}

\author{
Danthasinghe Waduge Badrajee Piyarathna, ${ }^{1,2}$ Akhila Balasubramanian, ${ }^{1,2,3}$ James M. Arnold, ${ }^{1,2}$ Stacy M. Lloyd, ${ }^{1,2}$ \\ Balasubramanyam Karanam, ${ }^{4}$ Patricia Castro, ${ }^{5,6}$ Michael M. Ittmann,, 5,6 Nagireddy Putluri, ${ }^{1,2}$ Nora Navone, ${ }^{7}$ Jeffrey A. Jones, ${ }^{8}$ \\ Wendong Yu, ${ }^{5}$ Vlad C. Sandulache, ${ }^{9}$ Andrew C. Sikora, ${ }^{9}$ George Michailidis, ${ }^{10}$ and Arun Sreekumar ${ }^{1,2,3}$

\begin{abstract}
DDepartment of Molecular and Cellular Biology, ${ }^{2}$ Alkek Center for Molecular Discovery, and ${ }^{3}$ Verna and Marrs McLean Department of Biochemistry and Molecular Biology, Baylor College of Medicine, Houston, Texas, USA. ${ }^{4}$ Department of Biology and Cancer Research, Tuskegee University, Tuskegee, Alabama, USA. ${ }^{5}$ Department of Pathology and ${ }^{6}$ Dan L. Duncan Comprehensive Cancer Center, Baylor College of Medicine, Houston, Texas, USA. 'Department of Genitourinary Medical Oncology, The University of Texas MD Anderson Cancer Center, Houston, Texas, USA. ${ }^{8}$ Michael E. DeBakey Veteran Affairs Medical Center and Department of Urology and ${ }^{9}$ Bobby R. Alford Department of Otolaryngology-Head and Neck Surgery, Baylor College of Medicine, Houston, Texas, USA. ${ }^{10}$ Department of Statistics, University of Florida, Gainesville, Florida, USA.
\end{abstract}

BACKGROUND. African American patients have higher cancer mortality rates and shorter survival times compared with European American patients. Despite a significant focus on socioeconomic factors, recent findings strongly argue the existence of biological factors driving this disparity. Most of these factors have been described in a cancer-type specific context rather than a pan-cancer setting.

METHODS. A novel in silico approach based on Gene Set Enrichment Analysis (GSEA) coupled to transcription factor enrichment was carried out to identify common biological drivers of pan-cancer racial disparity using The Cancer Genome Atlas data set. Mitochondrial content in patient tissues was examined using a multi-cancer tissue microarray approach (TMA).

RESULTS. Mitochondrial oxidative phosphorylation was uniquely enriched in tumors from African American patients compared with tumors of various cancer types from European American patients. Tumors from African American patients also showed strong enrichment for the ERR1-PCC1 $\alpha$-mediated transcriptional program, which has been implicated in mitochondrial biogenesis. TMA analysis revealed that cancers from African American patients harbor significantly more mitochondria compared with the same cancers from European American patients.

CONCLUSION. These findings highlight changes in mitochondria as a common distinguishing feature among tumors from African American versus European American patients in a pan-cancer setting, and provide the rationale for the repurposing of mitochondrial inhibitors to treat cancers from African American patients.

FUNDING. This research was partially supported by National Institutes of Health grants NIH U01 CA167234, NIH 1 U01 CA17967401A1, 5R01CM11402903, 1U01CA23548701, U01 CA167234, R01CA220297, and R01CA216426; pilot and shared resources support from Dan L. Duncan Cancer Center grant P30 CA125123; and NCI SPORE pilot grant NIH P50 CA186784. It was also partially supported by the Diana Helis Henry Medical Research Foundation; the Brockman Foundation; Agilent Technologies; Department of Defense grants W81XWH-12-1-0130 and W81XWH-12-1-0046; Cancer Prevention Research Institute of Texas grant RP120092; a Prostate Cancer Foundation Challenge Award; National Science Foundation grant DMS-1545277; and American Cancer Society grant 127430-RSG-15-105-01-CNE.

\section{Introduction}

African American cancer patients have higher mortality rates and shorter survival times compared with European American patients

\section{Related Commentary: p. 2187}

Authorship note: $A B$ and JMA contributed equally to this work Conflict of interest: The authors have declared that no conflict of interest exists. Copyright: (c) 2019, American Society for Clinical Investigation. Submitted: January 21, 2019; Accepted: March 12, 2019; Published: May 6, 2019 Reference information: J Clin Invest. 2019;129(6):2351-2356. https://doi.org/10.1172/JCl127579.
$(1-5)$. In the United States, for every 100,000 cancer patients, the age-adjusted cancer-associated mortality for African American versus European American patients was 189.54 and 163.54 respectively, resulting in a disparity ratio of $13.87 \%(2-5)$. When examined for individual tumor types, the disparity in mortality was higher in African American patients for cancers of the breast, colon, rectum, uterus, liver, lung, bronchus, and prostate with disparity ratios ranging from $6.41 \%$ (for lung cancer across both sexual phenotypes) to $118.52 \%$ (for men with prostate cancer [PCa]). This observation is consistent with the 5- and 10-year survival data obtained from the Surveillance Epidemiology and End Results database (Supplemental Table 1; supplemental material available online with this article; 
A

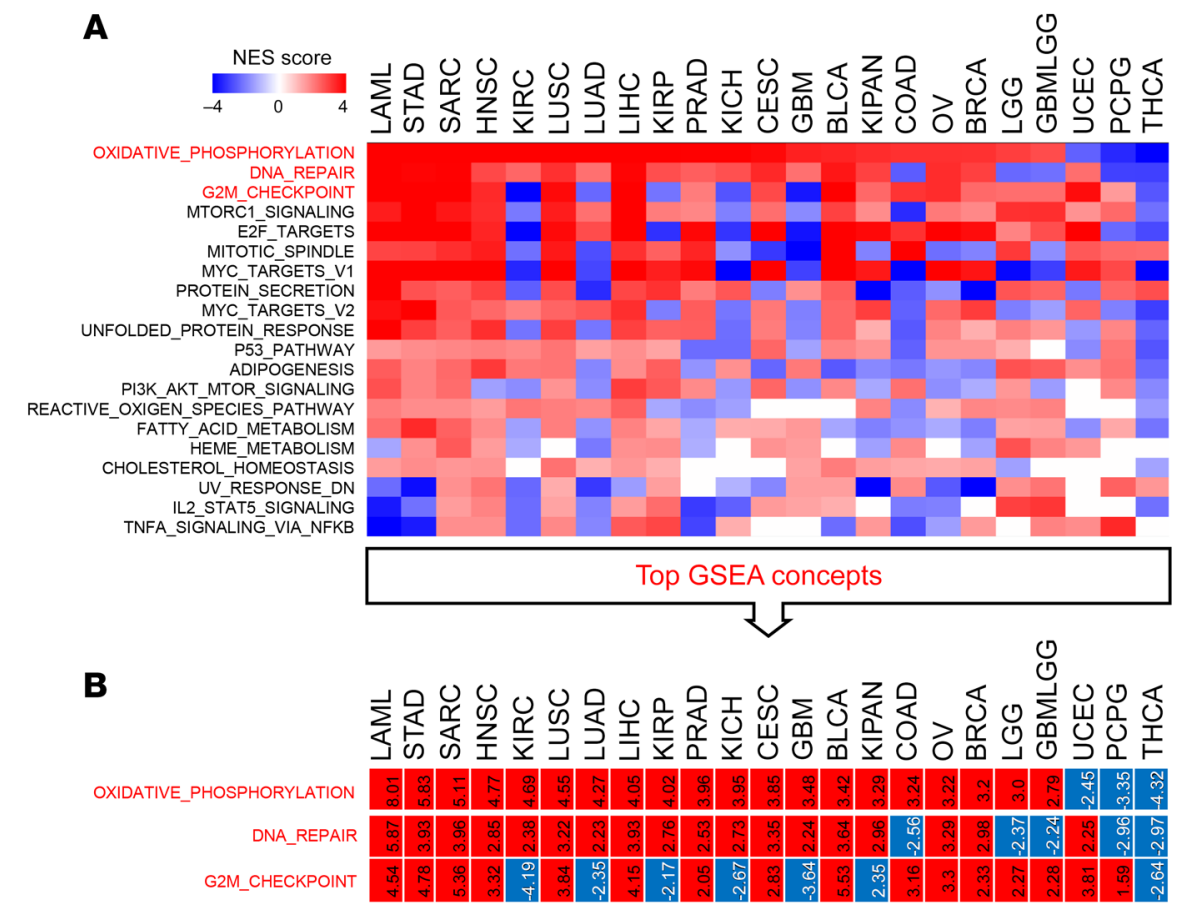

C

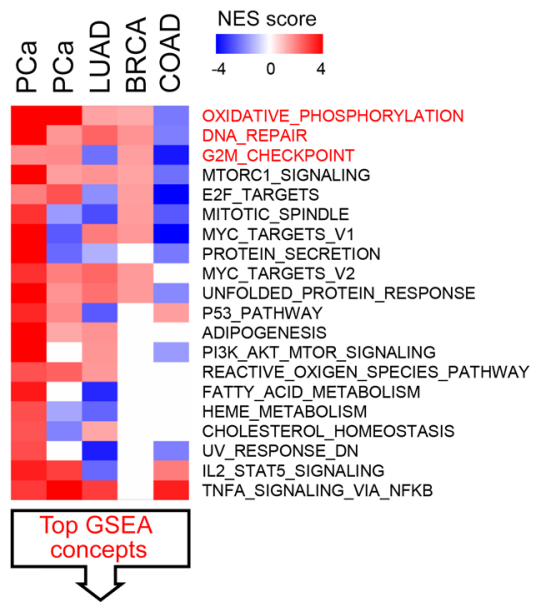

D

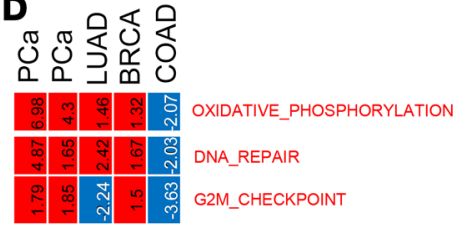

Figure 1. Molecular concepts significantly enriched in tumors from self-reported African American patients compared with tumors from European American patients in pan-cancer GSEA analysis. (A) Heatmap showing the top 20 commonly enriched pathways in tumors from African American patients compared with tumors from European American patients, across multiple cancers. See normalized enrichment score (NES) scale on the top. All GSEA concepts listed are significant at FDR $<0.25$. (B) Table showing the individual NES for the 3 top commonly enriched pathways for each cancer type (FDR < 0.05). (C) Heatmap showing enrichment of OXPHOS in 5 independent cancer data sets. (D) Table showing individual NES for the 5 independent data sets. Columns represent cancer type, and rows represent the enriched concepts. Shades of red and blue describe positive and negative enrichment scores, respectively. LAML, acute myeloid leukemia; STAD, stomach adenocarcinoma; SARC, sarcoma; HNSC, head and neck squamous cell carcinoma; KIRC, kidney renal clear cell carcinoma; LUSC, lung squamous cell carcinoma; LUAD, lung adenocarcinoma; LIHC, live hepatocellular carcinoma; KIRP, kidney renal papillary cell carcinoma; PRAD, prostate adenocarcinoma; $\mathrm{KICH}$, kidney chromophobe; CESC, cervical squamous cell carcinoma and endocervical adenocarcinoma; GBM, glioblastoma multiforme; BLCA, bladder cancer; KIPAN, pan-kidney cohort; COAD, colon adenocarcinoma; OV, ovarian adenocarcinoma; BRCA, breast cancer; LGG, brain low grade glioma; CBMLGG, glioblastoma multiforme low grade glioma; UCEC, uterine corpus endometrial carcinoma; PCPG, pheochromocytoma and paraganglioma; THCA, thyroid carcinoma; PCa, prostate cancer.

https://doi.org/10.1172/JCI127579DS1). The specific reasons for this disparity have not been identified. However, genetic/biologic, environmental, and health care access/utilization factors are all thought to be contributory (6).

Recent studies suggest that biological factors can be crucial for racial inequalities in cancer incidence and clinical outcomes $(7$, 8). For example, aggressive triple-negative breast cancer (BRCA) is more prevalent in African American women and is associated with higher levels of resistin, interleukin 6 (9), and 2-hydroxyglutarate (10). PCa in African American patients has higher levels of $17 \beta$-estradiol (11), prostate-specific antigen (12), androgen receptor expression (13), and altered mitochondria (14). African American patients with hepatocellular carcinoma have a 7-fold increase in apolipoprotein 1 (APOA1) expression and reduction in hepatocyte nuclear factor $4 \alpha(\mathrm{HNF} 4 \alpha)$, with the latter being associated with increased metastasis (15). Furthermore, African American patients with non-small cell lung cancer have higher circulating levels of IL-1 $\beta$, interleukin 10 (IL-10), and tumor necrosis factor $\alpha$ (TNF- $\alpha$ ) compared with European American patients (16). Taken together, although these findings demonstrate independent disease site-specific biological alterations among African American and European American patients, they fail to explain the pan- cancer disparity in tumor progression and clinical outcomes observed between these 2 populations. In light of this, our main goal was to examine if there exist common biological alterations across multiple cancers that distinguish tumors from African American patients from those of European American patients.

\section{Results}

We performed Gene Set Enrichment Analysis (GSEA) for 23 different cancer types independently using The Cancer Genome Atlas (TCGA) gene expression data, looking for common hallmark alterations capable of distinguishing tumors from African American patients from those of European American patients, across multiple cancer types (Figure 1A). These 23 cancer data sets contained data on at least 10 tumors from African American patients. GSEA revealed positive enrichment for concepts including oxidative phosphorylation (OXPHOS), DNA repair, and G2M checkpoint in tumors from African American patients versus those from European American patients, across multiple cancer types (Figure 1, A and B, Supplemental Table 2; $P<0.01, \mathrm{FDR}<25 \%$ ). Coenrichment of DNA repair and the G2M checkpoint is not surprising as both are related to preserving DNA fidelity (17). OXPHOS is associated with mitochondria and energy production, and the 


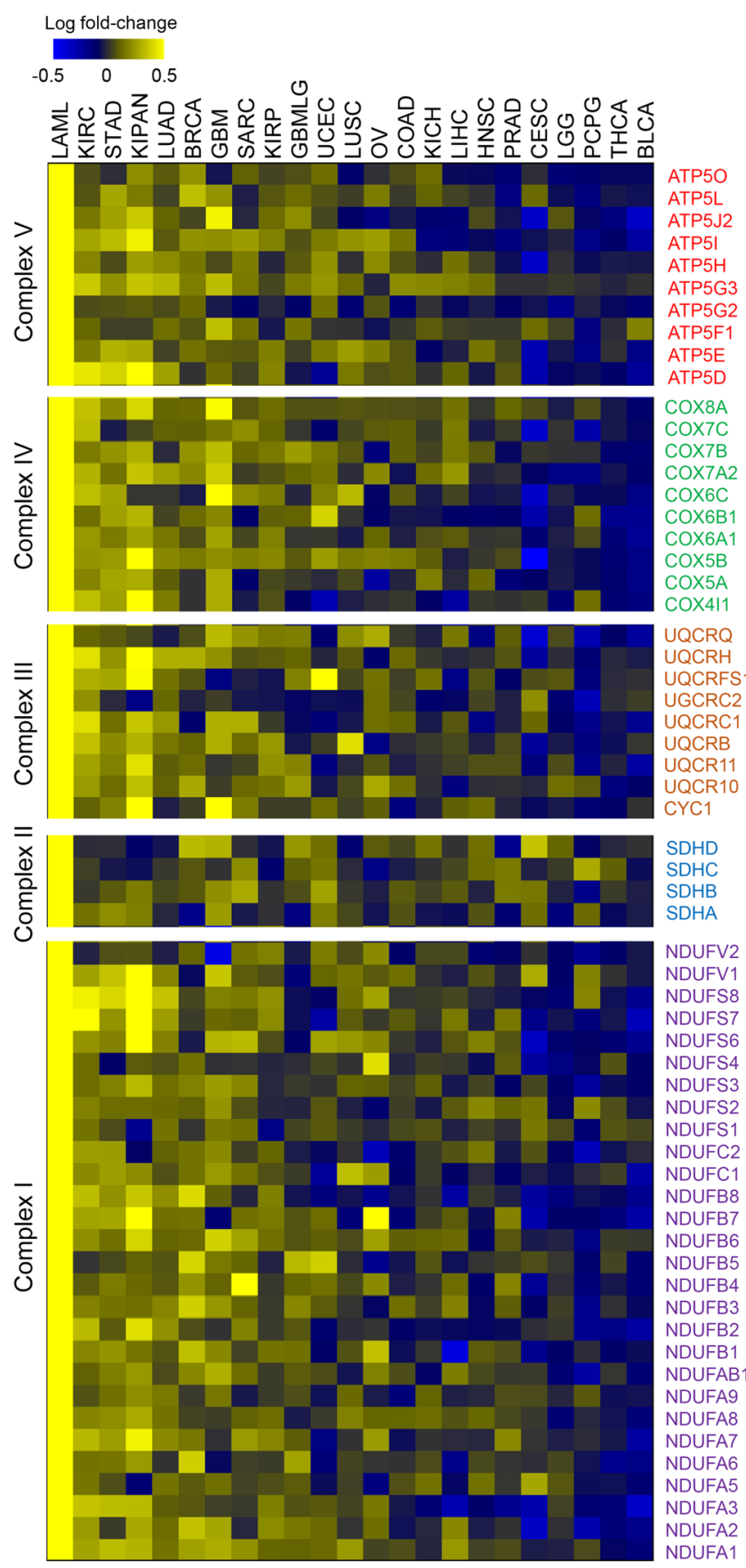

generation of DNA damaging reactive oxygen species (ROS) (18). To validate these initial findings, we performed GSEA on 5 independent data sets (GSE37751-BRCA, GSE64331-PCa, GSE6956PCa, GSE101929-lung cancer, GSE28000-colon cancer) and found a similar trend for OXPHOS enrichment in tumors from African American patients compared with tumors from European American (Figure 1, C and D, and Supplemental Table 3). Analysis of a subset of genes encoding different mitochondrial complexes within the enriched OXPHOS pathway demonstrated that these were ubiquitously upregulated in several types of tumors from African American patients (Figure 2, Supplemental Table 4, refer to Supplemental Figure 1 for heatmap containing top $50 \%$ of genes associated with OXPHOS concept). With this in mind, we
Figure 2. Genes associated with oxidative phosphorylation are elevated in tumors from African American patients relative to tumors from European American patients, across 23 cancer types in TCGA. Log fold-change in expression of the genes between African American and European American tumors is shown. Shades of yellow and blue describe increased and reduced fold-change in African American versus European American comparison, respectively. Columns contain different cancer types and rows contain the genes. Genes are grouped based on their membership in the 5 different mitochondrial complexes.

hypothesized that there exist common transcriptional regulators controlling the OXPHOS gene set in tumors from African American patients across different cancer types.

To identify common transcriptional regulators of the OXPHOS gene set, we performed transcription factor enrichment analysis on each of the 23 cancer data sets. Among the different transcription factors identified within each cancer type (representative example for BRCA in Figure 3A), estrogen-related receptor 1 (ERR1) was the most significant and commonly enriched transcription factor in tumors from African American patients across all the 23 cancer types (Figure 3B, $P<0.0001$, FDR $<0.01 \%$, Supplemental Table 5). ERR1 is known to regulate mitochondrial biogenesis in the presence of peroxisome proliferator-activated receptor gamma coactivator $1 \alpha$ (PGC1 $\alpha)$ (19). Indeed, we found that the 23 cancer types were also co-enriched for PGC1 $\alpha$ (Figure 3C). Additionally, there was a significant positive correlation between ERR1 and PGC1 $\alpha$ for these cancer types (Figure 3D, Supplemental Table 6, $P=0.02, r=0.47)$. Collectively, our findings suggest that tumors from African American patients are defined by elevated OXPHOS coupled to ERR1 and PGC1 $\alpha$ gene set enrichment.

Given that ERR1 and PGC1 $\alpha$ together have been shown to regulate mitochondrial biogenesis, and that OXPHOS is a product of mitochondrial respiration, we hypothesized that tumors from African American patients would possess more mitochondria compared with tumors from European American patients. To test this hypothesis, we performed tissue microarray analysis (TMA) on a cohort of PCa, laryngeal, and oral cancer samples, staining for mitochondria. Samples from African American patients stained significantly higher for mitochondria than those from European American patients in all 3 cancer types (Figure 4; refer to Supplemental Tables 7-9 for clinical data associated with the TMAs).

\section{Discussion}

Our data suggest that tumors from African American patients predominantly express more mitochondria, ERR1, and PCG1 $\alpha$ in multiple cancer types. These findings could form the biological basis of disparity in pan-cancer clinical outcomes seen in African American patients. Interestingly, clinical trial data for metformin, a mitochondrial inhibitor, has shown that African American patients, in general, respond better to this drug than European American patients (20). Metformin was also more effective in reducing the incidence of PCa and the risk of colorectal cancer death in African American patients compared with European American patients $(21,22)$. These findings provide a rationale for evaluating existing mitochondrial drugs to treat tumors from African American patients. Additionally, from a biomarker perspective, validation of our results could lead to the development 
A

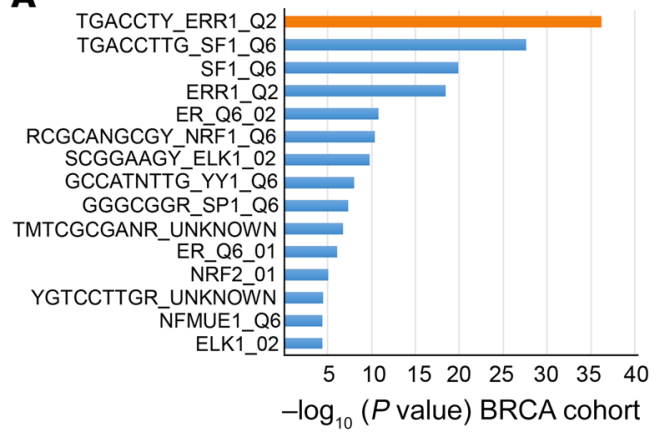

D

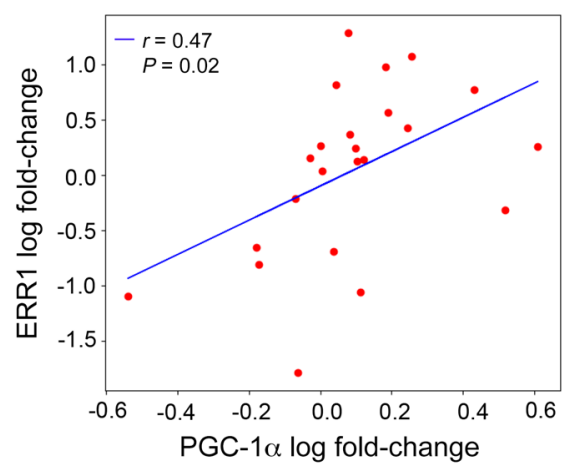

B

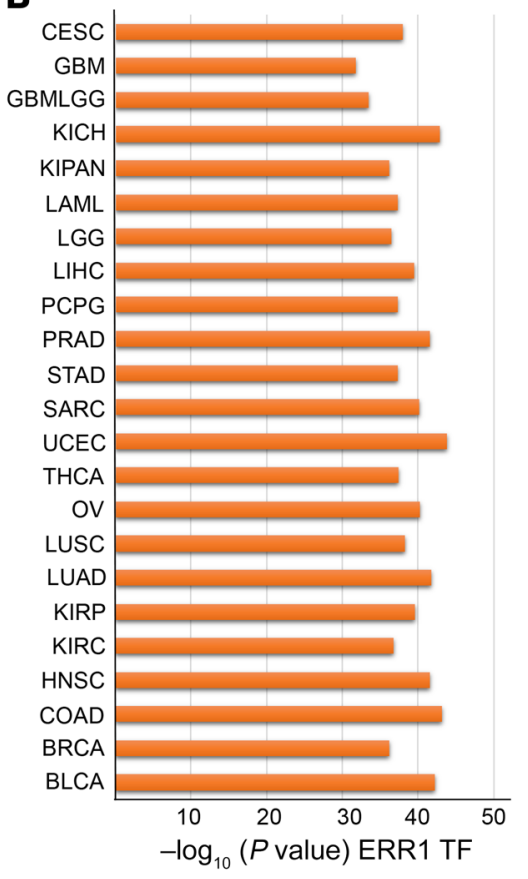

C

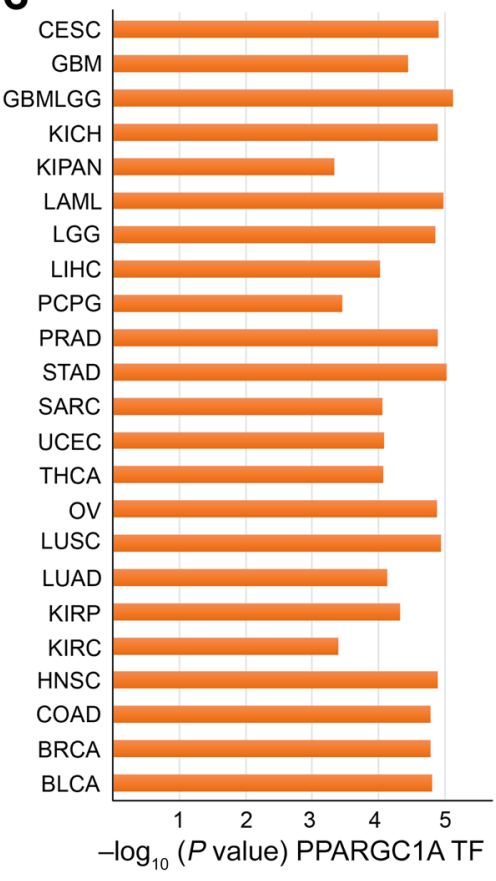

Figure 3. OXPHOS gene cluster in tumors from African American patients enriches for ERR1 and PGC1 $\alpha$ transcription factor motifs. (A) List of transcription factor motifs enriched by the OXPHOS cluster in breast cancer (BRCA). The significance of the enrichment is described in the $x$ axis. The $y$ axis describes the different transcription factor motifs. ERR1 is the most enriched transcription factor motif in the OXPHOS cluster in breast cancer. (B) ERR1 is significantly enriched in OXPHOS gene clusters across multiple cancers. The significance of the enrichment is described in the $x$ axis. The $y$ axis describes the different cancer types. (C) Same as in B, but for PGC1 $\alpha$. (D) A significant positive correlation is observed between ERR1 and PGC1 $\alpha$ expression in tumors from African American patients enriched for OXPHOS pathway across multiple cancers.

of mitochondrial metabolites as noninvasive biomarkers for cancer prognosis. Although this is the first report to demonstrate the existence of a common biological alteration in tumors from African American patients in a pan-cancer setting, there is a limitation that should be addressed in future studies. In our study, the stratification of patients into African American or European American groups in the TCGA data was solely based on self-reported information associated with the clinical files accompanying these data sets. It is essential to confirm these results using ancestry-verified tumor data from African American patients. We have addressed this partially by using ancestry-verified PCa TMA that confirms increased mitochondria in tumors from African American patients relative to tumors from European American patients. Irrespective of this caveat, we expect our studies to motivate mechanistic studies focused on mitochondria using ancestry-verified preclinical models of African American patients.

\section{Methods}

GSEA. To characterize biologically relevant changes in molecular signaling pathways among African American and European American patients, we employed GSEA (23) to identify significantly enriched concepts in each of the 23 tumor types in TCGA, each of which contained data from at least 10 African American patients. The procedure for GSEA involves determining whether a predefined set of genes (e.g., genes involved in a particular molecular signaling pathway) is significantly different between any 2 groups. The entire list of genes is ranked according to expression differences between 2 experimental conditions. An enrichment score for each gene set is then calculated. This score represents the extent of overrepresentation of a gene set at either end of the continuum. In GSEA, the cumulative distribution function was constructed by performing 1000 random gene set membership assignments. A nominal $P<0.01$ and a FDR $<25 \%$ were used as thresholds for determining the significance of the enrichment score (ES).The methodology works in synchrony with the Molecular Signatures Database (MSigDB), which provides the gene set definitions in the form of 8 major collections (13,311 total gene sets). Out of the 8 gene set collections, we focused on well-defined, large-scale biological processes, termed the Hallmark $(\mathrm{H})$ Gene Set. For the GSEA, self-reported data on race were used to stratify TCGA samples into African American and European American groups.

Enrichment analysis to identify key transcription factors regulating the OXPHOS gene cluster. The OXPHOS gene cluster was defined as the set of core genes that contributed to the enrichment of the OXPHOS pathway in GSEA. We performed an enrichment analysis using the hypergeometric method to identify transcription factors motifs (TFT, C3) in the OXPHOS gene set derived from the MSigDB for each cancer type. A nominal P $<0.0001$ and a FDR $<0.01 \%$ were used as thresholds to determine the significance of the enrichment. The results were represented using heat maps and bar graphs implemented using the $\mathrm{R}$ package.

TMA. The PCa TMA used for mitochondrial staining was built by the Pathology and Histology Core at the Baylor College of Medicine. 
A

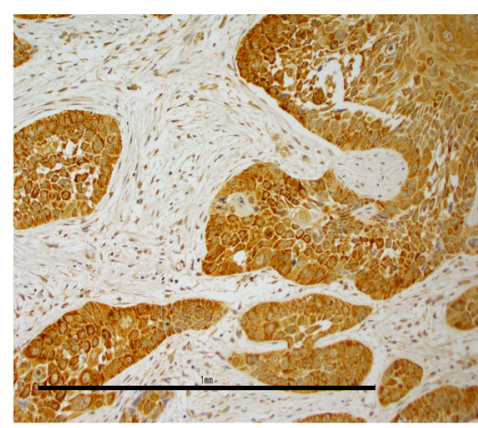

B

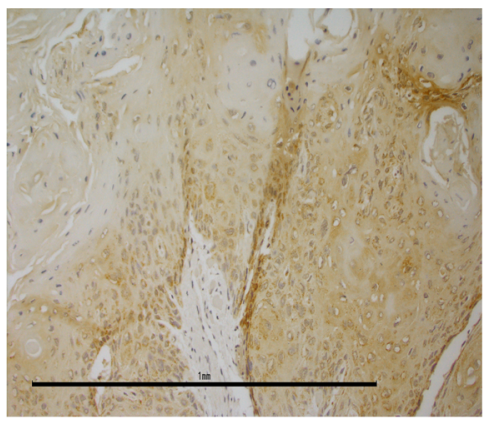

C

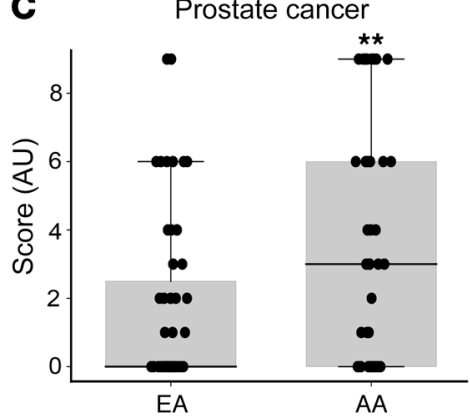

D

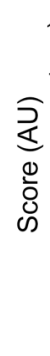

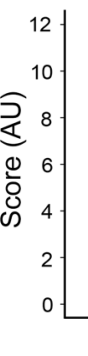

Laryngeal cancer

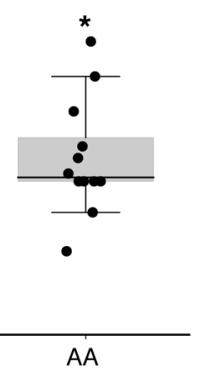

E

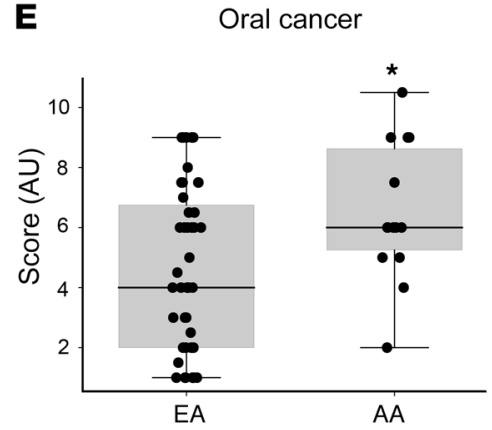

Figure 4. Tissue microarray analysis shows increased mitochondria in samples from African American patients. (A) Tissue microarray analysis (TMA) of samples stained for mitochondria showed increased mitochondrial staining in African American patients compared with (B) European American patients (representative image). Scale bars: $1 \mathrm{~mm}$. (C) TMA data showing increased staining for mitochondria in prostate cancer in African American patients ( $n=$ 53) compared with prostate cancer in European American patients $(n=51)$. (D) Same as in C, but for laryngeal cancer $(n=12$ African American patients; $n=$ 17 European American patients). (E) Same as in C, but for oral cancer ( $n=14$ African American patients; $n=43$ European American patients). ${ }^{* *} P<0.01$, ${ }^{*} P<0.05$; Wilcoxon rank sum test; data are mean \pm SD.

This TMA comprised 53 stainable ancestry-verified tissue sections from African American patients and 51 stainable tissue sections from European American patients. Laryngeal and oral cancer TMAs containing samples from self-reported African American patients were obtained from Vlad C. Sandulache and Andrew Sikora. The laryngeal cancer TMA comprised 11 stainable tissue sections from African American patients and 18 stainable tissue sections from European American patients. The oral cancer TMA comprised 14 stainable tissue sections from African American patients and 43 stainable tissue sections from European American patients.

The tissues were deparaffinized using xylene and rehydrated using a graded alcohol series. The slides were pressure cooked for 10 minutes to retrieve mitochondrial antigens. The slides were then treated with $3 \%$ hydrogen peroxide for 5 minutes to quench endogenous peroxidase activity. Slides were blocked with $3 \%$ goat serum at room temperature for 1 hour in a humidity chamber before staining with an anti-mitochondrial antibody (1:100 dilution of mouse monoclonal antibody, Abcam ab92824). The secondary antibody was applied for 40 minutes (HRP-conjugated goat anti-mouse secondary antibody, Jackson Immunoresearch Laboratories). Diaminobenzidine was applied for 7 minutes to visualize the antigen-antibody reaction, and the slides were counterstained with hematoxylin for 1 minute. Each staining run included positive and negative controls; negative controls were obtained by omitting the primary antibody. Slides were then dehydrated in an alcohol series and cleared in xylene baths before being mounted with Permount media (Sigma-Aldrich). The PCa TMA was scored by Michael Ittmann, a genitourinary pathologist. The laryngeal and oral cancer TMAs were scored by Wendong Yu, a head and neck cancer pathologist. The TMA staining was determined using a combination of an intensity and extent score. These 2 values were then multiplied to generate a final score that was used to make the box plots.

Statistics. $P$ values for the enrichment analyses were generated through the GSEA permutation test (1000 permutations). The FDR was determined using the Benjamini-Hochberg procedure (24). Significance for the mitochondrial TMA analysis was determined using a 2-tailed Wilcoxon rank sum test. A $P$ value less than 0.05 was considered significant.

Study approval. The use of all human tissues in this study was reviewed and approved by the Institutional Review Board at Baylor College of Medicine.

\section{Author contributions}

DWBP and AS formulated and designed the study. DWBP, JMA, and $\mathrm{AB}$ performed the bioinformatics analyses. JMA, $\mathrm{AB}$, and SML wrote the manuscript. $\mathrm{AB}$ performed the in vitro studies. SML analyzed TMA staining data. BK performed TMA staining. PC provided TMA slides. MMI and WY performed TMA scoring. NP and NN provided scientific input. JAJ performed clinical sample annotation. VCS and AGS provided the TMAs. GM reviewed the statistical analyses. All authors reviewed the manuscript.

\section{Acknowledgments}

The authors acknowledge the joint participation of the Diana Helis Henry Medical Research Foundation through its direct 
engagement in the continuous active conduct of medical research in conjunction with the Baylor College of Medicine (BCM) and the Global Center for Mass Spectrometry Excellence supported by Agilent Technologies at BCM. This research was partially supported by National Institutes of Health grants NIH U01 CA167234 (to AS and GM), NIH 1 U01 CA17967401A1 and U01 CA167234 (to AS), 5R01GM11402903 and 1U01CA23548701 (to GM), R01CA220297 and R01CA216426 (to NP); Dan L. Duncan Cancer Center grant P30 CA125123 (supporting a pilot project [to AS] and the Metabolomics Core and the Human Tissue Acquisition and Pathology Core [to AS and MI]); and NCI SPORE pilot grant NIH P50 CA186784 (to AS). It was also partially supported by Department of Defense grants
W81XWH-12-1-0130 (to AS and GM) and W81XWH-12-1-0046 (to MI); Cancer Prevention Research Institute of Texas grant RP120092 (to AS); a Prostate Cancer Foundation Challenge Award (to AS, MI, JAJ, and NN); a Brockman Foundation and Agilent Technologies grant (to AS); National Science Foundation grant DMS-1545277 (to GM); and American Cancer Society grant 127430-RSG-15-105-01-CNE (to NP).

Address correspondence to: Arun Sreekumar, Department of Molecular and Cell Biology and Verna and Marrs McLean Department of Biochemistry and Molecular Biology, Baylor College of Medicine, 120D, Jewish Building, Houston, Texas, 77030, USA. Phone: 713.798.3305; Email: sreekuma@bcm.edu.
1. O'Keefe EB, Meltzer JP, Bethea TN. Health disparities and cancer: racial disparities in cancer mortality in the United States, 2000-2010. Front Public Health. 2015;3:51.

2. Jemal A, Bray F, Center MM, Ferlay J, Ward E, Forman D. Global cancer statistics. CA Cancer J Clin. 2011;61(2):69-90.

3. Kamangar F, Dores GM, Anderson WF. Patterns of cancer incidence, mortality, and prevalence across five continents: defining priorities to reduce cancer disparities in different geographic regions of the world. J Clin Oncol. 2006;24(14):2137-2150.

4. Jemal A, Center MM, DeSantis C, Ward EM. Global patterns of cancer incidence and mortality rates and trends. Cancer Epidemiol Biomarkers Prev. 2010;19(8):1893-1907.

5. Thun MJ, DeLancey JO, Center MM, Jemal A, Ward EM. The global burden of cancer: priorities for prevention. Carcinogenesis. 2010;31(1):100-110.

6. Powell IJ. Epidemiology and pathophysiology of prostate cancer in African-American men.JUrol. 2007;177(2):444-449.

7. Wallace TA, Martin DN, Ambs S. Interactions among genes, tumor biology and the environment in cancer health disparities: examining the evidence on a national and global scale. Carcinogenesis. 2011;32(8):1107-1121.

8. Daly B, Olopade OI. A perfect storm: How tumor biology, genomics, and health care delivery patterns collide to create a racial survival disparity in breast cancer and proposed interventions for change. CA Cancer J Clin. 2015;65(3):221-238.

9. Deshmukh SK, et al. Resistin and interleukin-6 exhibit racially-disparate expression in breast cancer patients, display molecular association and promote growth and aggressiveness of tumor cells through STAT3 activation. Oncotarget. 2015;6(13):11231-11241.

10. Terunuma A, et al. MYC-driven accumulation of 2-hydroxyglutarate is associated with breast cancer prognosis. J Clin Invest. 2014;124(1):398-412.

11. Abd Elmageed ZY, et al. High circulating estrogens and selective expression of ER $\beta$ in prostate tumors of Americans: implications for racial disparity of prostate cancer. Carcinogenesis. 2013;34(9):2017-2023.

12. Moul JW, et al. Prostate-specific antigen values at the time of prostate cancer diagnosis in AfricanAmerican men. JAMA. 1995;274(16):1277-1281.

13. Hatcher D, Daniels G, Osman I, Lee P. Molecular mechanisms involving prostate cancer racial disparity. Am J Transl Res. 2009;1(3):235-248.

14. Xiao J, Cohen P, Stern MC, Odedina F, Carpten J, Reams R. Mitochondrial biology and prostate cancer ethnic disparity. Carcinogenesis. 2018;39(11):1311-1319.

15. Ning BF, Liu J, Xu WP, Yin C, Zhang X, Xie WF. HNF $4 \alpha$ inhibits liver cancer metastasis via suppression of NF-кB activity. Proc 105th Anпи Meet Am Assoc Cancer Res. 2014;74(19 Suppl):Abstract 134. http://cancerres.aacrjournals.org/content/74/19_Supplement/134.

16. Pine SR, et al. Differential serum cytokine levels and risk of lung cancer between African and European Americans. Cancer Epidemiol Biomarkers Prev. 2016;25(3):488-497.

17. Lukas J, Lukas C, Bartek J. Mammalian cell cycle checkpoints: signalling pathways and their organization in space and time. DNA Repair (Amst). 2004;3(8-9):997-1007.

18. Chen Q, Vazquez EJ, Moghaddas S, Hoppel CL, Lesnefsky EJ. Production of reactive oxygen species by mitochondria: central role of complex III. J Biol Chem. 2003;278(38):36027-36031.

19. Park S, et al. ERR $\alpha$-regulated lactate metabolism contributes to resistance to targeted therapies in breast cancer. Cell Rep. 2016;15(2):323-335.

20. Williams LK, et al. Differing effects of metformin on glycemic control by race-ethnicity. JClin Endocrinol Metab. 2014;99(9):3160-3168.

21. Feng T, et al. Metformin use and risk of prostate cancer: results from the REDUCE study. Cancer Prev Res (Phila). 2015;8(11):1055-1060.

22. Zhu RC, et al. Survival benefits in colorectal adenocarcinoma with the use of metformin among a black diabetic inner city population. Colorectal Cancer. 2017;6(1):33-41.

23. Subramanian A, et al. Gene set enrichment analysis: a knowledge-based approach for interpreting genome-wide expression profiles. Proc Natl Acad Sci U S A. 2005;102(43):15545-15550.

24. Benjamini Y, Hochberg Y. Controlling the false discovery rate: a practical and powerful approach to multiple testing. J R Stat Soc Ser B. 1995;57(1):289-300. 